\title{
VITULIA IVONE: JUSTICIA REPRODUCTIVA: LA INTERUPCIÓN DEL EMBARAZO Y LA OBJECIÓN DE CONCIENCIA EN ITALIA'.
}

\author{
REPRODUCTIVE JUSTICE: ABORTION AND CONSCIENTIOUS OBJECTION IN \\ ITALY.
}

RESUMEN: El tema de la salud - que encuentra su protección en la Constitución italiana - es relacionado con el derecho-deber de información sanitaria. Entre los derechos reproductivos, la visión del aborto como elección consciente e informada relega la cuestión de la objeción de conciencia - que lamentablemente planea problemas concretos de peligro de vida para las mujeres - en el solo caso de infracción de un servicio.

ABSTRACT: The issue of Health - which finds its protection in the Italian Constitution - is related to the right-duty of health information. Among reproductive rights, the vision of abortion as a conscious and informed choice relegates the issue of conscientious objection - which unfortunately devises specific life-threatening problems for life's women in the only case of a public service disrupsion.

PALABRAS CLAVE: salud - aborto - justicia

KEY WORDS: Health - Abortion - Justice

\section{EL DERECHO A LA SALUD COMO DERECHO SOCIAL.}

La salud es un valor fundamental planeado en la Constitución italiana, en el art. 32 que afirma: «La República protege la salud como derecho fundamental de la persona y interes colectivo, y garantiza la atención médica gratuita a los indigentes.

Nadie puede ser obligado a un tratamiento médico específico a menos que lo requiera la ley. La ley no puede en ningún caso violar los límites impuestos por el respeto a la persona humana».

El primer párrafo da a la salud la calificación de derecho "fundamental"2 caracterizado por dos funciones principales, es decir el derecho del individuo y el interés

\footnotetext{
${ }^{1}$ Profesora de Derecho Privado. Dipartimento di Scienze Giuridiche (Scuola di Giurisprudenza). Università di Salerno. vituliaivone@unisa.it http://www.unisa.it/docenti/vituliaivone/index

${ }^{2}$ V. CRISAFULLI, Le norme programmatiche della Costituzione, 1952; P. BARILE, Diritti dell'uomo e libertà fondamentali, Bologna, 1981; A. PACE, Problematica delle libertà costituzionali. Parte generale, Padova, 1992; C. AMIRANTE, Diritti di libertà e diritti sociali, Napoli, 1995; L. CARLASSARE, Forma di stato e diritti fondamentali, in Quaderni costituzionali, vol.1, 1995; F. MODUGNO, I «nuovi diritti» nella giurisprudenza costituzionale, Torino, 1995; R. GUASTINI, Specificità dell'interpretazione costituzionale?, Analisi e diritto, Torino, 1996; A. BALDASSARRE, Diritti della persona e valori costituzionali, Torino, 1997; P. BILANCIA - G. F. PIZZETTI, Aspetti e problemi del costituzionalismo multilivello, Milano, 2004; P. PERLINGIERI, La persona e i suoi diritti. Problemi del diritto civile, Napoli, 2005; S. LABRIOLA, (a cura di), Valori e principi del regime repubblicano. 3. Legalità e garanzie, Roma-Bari, 2006, p.451 ss.; Id, Piccola storia dell'attualità della Costituzione, Milano, 2006, 4, p.670 ss.
} 
colectivo: estos dos aspectos están estrechamente relacionados, y afectan la dimensión real del derecho a la salud en el sistema italiano de normas.

En el primer caso, se configura como un derecho a recibir beneficios, sino también como un estado de libertad - al menos en los países con un alto progreso científico - que se amplia a las opciones a eligir o no datas técnicas terapéuticas ${ }^{3}$.

En el segundo aspecto, el interés de los Estados a crear y mantener un adecuado estado de salud de su población ha crecido a lo largo de las décadas, lo que resulta en las políticas de salud ad hoc que representan una de las herramientas típicas utilizadas por un Estado para el ejercicio de la función de gobierno sobre las comunidades humanas situadas en su territorio.

Políticas de salud adecuadas permiten a un Estado en perseguir fines económicos que ayudan a satisfacer sus necesidades: una población sana, necesita de menos cuidados, reduce la incidencia de los gastos en salud sobre el presupuesto público; por otra parte, estos políticas también están dirigidas a limitar los daños a la salud pública que pueden ser el resultado de un bajo nivel de seguridad de los alimentos, de los actos de bioterrorismo o del uso de sustancias nocivas para la salud humana y animal.

El concepto de salud no es absoluto y definitivo, sino conectado y dependiente de corolarios importantes como la capacidad de la persona en conseguirla, la capacidad de planear opciones de vida, la capacidad / posibilidad de uso de la salud, de recursos y experiencias, de contexto ambiental donde la persona vive, de condiciones socioeconómicas específicas ${ }^{4}$.

El apartado 2 del art. 32 establece que "Nadie puede ser obligado a un tratamiento médico específico menos que sea requerido por la ley. La ley no puede en ningún caso violar los límites impuestos por el respeto a la persona humana". De este norma resulta el principio del consentimiento informado como regla ${ }^{5}$ : esta expresión habla del derecho del

${ }^{3}$ D. MORANA, La salute nella Costituzione italiana, Milano, 2002.

${ }^{4}$ L. CHIEFFI, (a cura), Il diritto alla salute alle soglie del terzo millennio. Profili di ordine etico, giuridico ed economico, Torino, 2003, p.125.

${ }^{5}$ F. MANTOVANI, II consenso informato: pratiche consensuali, in Riv.it.med.leg., 2000; M.C. VENUTI, Gli atti di disposizione del corpo, Milano, 2002; S. CACACE, Informazione, consenso e rifiuto di cure. (il)liceità del trattamento sanitario, in G. Comande' (a cura di), Diritto privato europeo e diritti fondamentali, Torino, 2004; C.M. D’ARRIGO, II contratto e il corpo: meritevolezza e liceità degli atti di disposizione dell'integrità fisica, in Familia, 2005; G. ALPA, II principio di autodeterminazione e le direttive anticipate sulle cure mediche, in Riv.crit.dir.priv., 2006; G. FACCI, Violazione del dovere di informazione da parte del sanitario e risarcimento del danno, in Resp.civ. e prev., 2006; G. FERRANDO, Stato vegetativo permanente e sospensione dei trattamenti medici, in Testamento biologico. Riflessioni di dieci giuristi, Fondazione Umberto Veronesi, 2006; A. PINNA, Autodeterminazione e consenso: da regola per i trattamenti sanitari a principio generale, in Contr.e impr., 2006; S. RODOTA', La vita e le regole. Tra diritto e non diritto, Milano, 2006; L. VIOLINI, A. OSTI, Le linee di demarcazione della vita umana, in M. Cartabia (a cura di), I diritti in azione. Universalità e pluralismo dei diritti fondamentali nelle Corti europee, Bologna, 2007, p.185 ss.; P. ZATTI, II corpo e la nebulosa dell'appartenenza, in Nuova giur.civ.comm., 2007; G. CRICENTI, I diritti sul corpo, Napoli, 2008; L. D'AVACK, Sul consenso informato all'atto medico, in II dir.fam. e pers., 2008; D. MESSINETTI, L'autodeterminazione dispositiva della persona e il valore della libertà del soggetto, in Riv.crit.dir.priv., 2008; G. MONTANARI VERGALLO, II rapporto medico paziente. Consenso e informazione tra libertà e responsabilità, Milano, 2008; G.U. RESCIGNO, Dal diritto di rifiutare un determinato trattamento sanitario secondo l'art.32, co. $2^{\circ}$ Cost., al princip io di autodeterminazione intorno alla propria vita, in Dir.pubbl., 2008; D. CARUSI, Tutela della salute, consenso alle cure, direttive anticipate: l'evoluzione del pensiero privatistico, in Riv.crit.dir.priv., 2009; C. CASONATO, Introduzione al biodiritto, Torino, 2009; T. 
DOI: http://dx.doi.org/10.12795/IETSCIENTIA.2017.i01.13

paciente, por un lado, de ser informado correctamente sobre términos, condiciones y consecuencias de la terapia que se le propone, y por otro, a aceptar libremente o rechazar el tratamiento propuesto. A pesar de su clara base constitucional, en Italia el principio del consentimiento informado en el tratamiento médico comenzó en realidad a impactar en las modalidades de la prestación de los servicios de salud sólo a partir de los años '90. En ese periodo, además de una serie de disposiciones que -aunque de manera fragmentadahan explicado este principio, se han multiplicado también las decisiones de los tribunales que han reconocido un verdadero "derecho al consentimiento informado".

En los últimos años el consentimiento informado ${ }^{6}$, necesario para considera legítimo cualquier tratamiento de salud, se realiza no sólo en la facultad de elegir entre varias terapias alternativas posibles, sino también a la de rechazar cualquier terapia y la de decidir su interrupción, incluso en presencia de un peligro para la salud, no importa aunque sea potencial o real ${ }^{7}$.

PASQUINO, Autodeterminazione e dignità della morte. Saggio di diritto civile, Padova, 2009; B. SALVATORE, Per uno studio sul consenso informato, in Dir.giur., 2009; U. CARNEVALI, Omessa informazione da parte del medico, danno da trattamento terapeutico e ipotetica scelta del paziente, in Resp.civ.e prev., 2010; C. CASTRONOVO, Autodeterminazione e diritto privato, in Europa e diritto privato, 2010; G. MARINI, II consenso, in Trattato di biodiritto, diretto da S. Rodotà e P.Zatti, Ambito e fonti del biodiritto, a cura di S.Rodotà e M.Tallacchini, Milano, 2010; F.D. BUSNELLI, Problemi giuridici di fine vita tra natura ed artificio, in Riv.dir.civ., 2011; G. CRICENTI, Diritto all'autodeterminazione? Bioetica dell'autonomia privata, in Nuova giur.civ.comm., 2011; G.RESTA, La disposizione del corpo. Regole di appartenenza e di circolazione, in Trattato di biodiritto, diretto da S.Rodotà e P.Zatti, Il governo del corpo, I, Milano, 2011; S. STEFANELLI, Autodeterminazione e disposizioni sul corpo, Perugia, 2011; E. CAVASINO, La flessibilità del diritto alla salute, Napoli, 2012; S. RODOTA', Il diritto di avere diritti, Roma-Bari, 2012; V. IVONE, Vulnerabilità del corpo e diritto al consenso, Napoli, 2013.

${ }^{6}$ S. PUGLIATTI, L'atto di disposizione e il trasferimento dei diritti, in Diritto civile, Metodo, teoria, Pratica. Saggi, Milano, 1951; M. PARADISO, II dovere del medico di informare il paziente. Consenso contrattuale e diritti della persona, in A.A.V.V., La responsabilità medica, Milano, 1982; F. GALGANO, Contratto e responsabilità contrattuale nell'attività sanitaria, in Riv. trim. dir. proc. civ., 1984, p.721; M. COSTANZA, Informazione del paziente e responsabilità del medico, in Giust.civ., 1986; L. ROSSI CARLEO, Brevi considerazioni sulla problematica della forma del consenso negli atti di disposizione del corpo, in La forma degli atti di diritto privato. Studi in onore di M.Giorgianni, Napoli, 1988; G. GRISI, L'obbligo precontrattuale di informazione, Napoli, 1990; R. ROMBOLI, Limiti alla libertà di disporre del proprio corpo nel suo aspetto "attivo" e in quello "passivo", in Foro it., 1991; R. DE MATTEIS, La responsabilità medica: un sottosistema della responsabilità civile, Padova, 1995; A. SANTOSUOSSO, Il consenso informato, Milano, 1996; C. CASTRONOVO, Profili della responsabilità medica, in Studi in onore di Pietro Rescigno, V, Milano, 1998, p.125 e segg.; G. FERRANDO, Consenso informato del paziente e responsabilità del medico. Principi, problemi e linee di tendenza, in Studi in onore di Pietro Rescigno, cit., 199 e segg. e spec. 231 e segg.; A. DONATI, Consenso informato e responsabilità da prestazione medica, in Rass.dir.civ., 2000.

El consentimiento libre e informado, según la Carta de Niza, encuentra su reconocimiento definitivo como expresión del derecho a la integridad humana, a ser protegidos en el campo de la medicina y la biología (art. 3, párr. 2). La norma se coloca en el capítulo I, titulado "Dignidad", que contiene cinco artículos, dedicados a la dignidad humana (Art. 1), al derecho a la vida (Art. 2), al derecho a la integridad de la persona (art. 3), a la prohibición de la tortura y de los tratos o penas inhumanos o degradantes (art. 4) y a la prohibición de la esclavitud y del trabajo forzoso (art. 5). Se vean M. CARTABIA, I diritti fondamentali in Europa dopo Lisbona: verso nuovi equilibri?, in Giornale dir. amm., 2010,p. 221; V. SCALISI, Ermeneutica dei diritti fondamentali e principio "personalista" in Italia e nell'Unione Europea, in Riv. dir. civ., 2010, p.157; S. NEGRI, Transplant Ethics and the International Crime of Organ Trafficking, in International Criminal Law Review, 1, 2016, p.1-17.

${ }^{7}$ Corte d'Appello Cagliari, sezione civile, (decr.), 16 gennaio 2009: «La autodeterminación de la persona, como regla general, se expresa en la Constitución, no es en absoluto incompatible con el derecho a la vida, que no está disponible en su título, pero no puede ser tan libre en los actos por los cuales se manifiesta su 
DOI: http://dx.doi.org/10.12795/IETSCIENTIA.2017.i01.13

\section{DESDE LA CONSTITUCIÓN ITALIANA HASTA LA LEY 833.}

Aunque desde 1948 la Constitución italiana regula el derecho a la salud como un derecho humano fundamental, la protección "debida" de la República ha quedado vinculada estrechamente al modelo de la seguridad social, en el que las responsabilidades públicas eran muy limitatas.

La Ley de 23 de diciembre de 1978, N833, ha estab lecido el Servicio Nacional de Salud. Junto con ello, el mismo año se aprobó la Ley $N^{\circ} 180$, sobre la protección de la salud mental, tan importante por sus principios y valores éticos y sociales. Sobre la base de lo que se expresa en el art. 32 de la Constitución, que protege el derecho a la salud como un principio básico para el interés individual y colectivo, la Ley $\mathrm{N}^{\circ} 833 \mathrm{crea}$ el Servicio Nacional de Salud, que se define como un complejo de funciones, servicios y actividades de promoción, mantenimiento y recuperación de la salud física y mental de toda la población, sin distinción de ningún tipo. Los principios presentes en la Ley son la totalidad de las intervenciones en materia de prevención, atención y rehabilitación; la igualdad de los ciudadanos en relación con el Servicio; la unidad de acción entre las instituciones públicas y privadas cuyas actividades van a afectar la salud de los ciudadanos; la participación de los ciudadanos en la aplicación del servicio a través de las formas de participación, para garantizar un control no institucional sobre la eficiencia y la eficacia de los diferentes niveles de intervención. Entre los objetivos están la superación de los desequilibrios regionales en las condiciones de salud del país a través de la planificación sanitaria adecuada y una distribución coherente de los recursos disponibles; la educación para la salud de los ciudadanos y las comunidades; la prevención de enfermedades y accidentes en todas las áreas de la vida y el trabajo; la seguridad en el empleo, con la participación de los trabajadores y de los sindicatos; el diagnóstico y el tratamiento de eventos de enfermedad cualesquiera que sean sus causas, la fenomenología y la duración de la discapacidad y la rehabilitación de la discapacidad; la atención de la salud mental; la procreación responsable y la protección de la maternidad y la infancia; la disciplina de las pruebas, la producción, la comercialización y distribución de drogas; promover y salvaguardar la salud y la higiene de la vida natural y el trabajo, etc.

Esta ley aplica las disposiciones constitucionales: en primer lugar el respeto de la dignidad y libertad de la persona humana, que representan tanto el fundamento que el objetivo final del derecho a la salud; a continuación, la universalidad de los destinatarios de los servicios, que son, por lo tanto, dirigidos a todos, no sólo a los trabajadores y sus familias; a continuación, insiste y ahonda en destacar la equidad y la igualdad en el acceso a los servicios, independientemente de las condiciones socioeconómicas y la ubicación en el territorio nacional. Por último, la integridad de las prestaciones y el derecho-deber a ser informado.

\footnotetext{
ejercicio. De ello se desprende que, en el caso de que un paciente està inconsciente y es portador de fuertes convicciones éticas y religiosas, como es el caso de los Testigos de Jehová, debe ser excluido por esa sola razón que debe someterse a tratamiento médico contra su fe. Sin embargo, es innegable, en tal caso, la necesidad de expresar el disenso al tratamiento a la transfusión sea o el mismo paciente que trayga consigo una, puntual, declaración expresa de manera inequívoca de la que emerge el deseo de evitar la transfusión, incluso en los casos de amenaza para la vida, o sea una persona diferente da él mismo indicada, que demostra la existencia de su poder representativo en esa medida y confirma esa disidencia cuando recibe las informaciones por los profesionales de la salud».
} 


\section{EL DERECHO-DEBER DE INFORMACIÓN SANITARIA.}

En la actualidad, el derecho-deber de información sanitaria juega un papel crucial en las relaciones médico-paciente, en especial, de cara a la interrupción voluntaria del embarazo, donde la información es condición indispensable para la validez del acto médico ${ }^{8}$.

En todo tratamiento o intervención médica respetuosa del principio de la autonomía de la voluntad del paciente hay que garantizar el derecho a la información jurídicosanitaria del que son titulares todos los ciudadanos, pero sin duda es en el terreno de la IVE donde este mandato se hace más necesario que nunca por los importantes intereses en juego que aquí se encierran.

Tanto en el ámbito nacional, como supranacional se va extendiendo una cultura de máximo respeto a la persona y su dignidad, sobre todo en lo que pertenece a su salud, entendida no como la ausencia de enfermedad y / o discapacidad física / psíquica, sino como un estado de completo bienestar físico, mental y social ${ }^{9}$.

En el marco regulador europeo y en la acción comunitaria, la persona humana ha adquirido una tarea central: tras la entrada en vigor del Tratado de Lisboa, el reformado art.6, par. 1 del Tratado de la UE confiere a la Carta «el mismo valor jurídico que los Tratados", consagrando así el carácter vinculante de los derechos fundamentales establecidos en este documento. El tema de la dignidad humana está presente en la

\footnotetext{
8 «La autodeterminación de la persona, como regla general, se expresa en la Constitución italiana, no es en absoluto incompatible con el derecho a la vida, que no está disponible en su título, pero no puede ser tan libre en los actos por los cuales se manifiesta su ejercicio. De ello se desprende que, en el caso de que un paciente està inconsciente y es portador de fuertes convicciones éticas y religiosas, como es el caso de los Testigos de Jehová, debe ser excluido por esa sola razón que debe someterse a tratamiento médico contra su fe. Sin embargo, es innegable, en tal caso, la necesidad de expresar el disenso al tratamiento a la transfusión sea o el mismo paciente que trayga consigo una, puntual, declaración expresa de manera inequívoca de la que emerge el deseo de evitar la transfusión, incluso en los casos de amenaza para la vida, o sea una persona diferente da él mismo indicada, que demostra la existencia de su poder representativo en esa medida y confirma esa disidencia cuando recibe las informaciones por los profesionales de la salud». Asì, Corte d'Appello Cagliari, sezione civile, (decr.), 16 gennaio 2009.

${ }^{9}$ Tal como se define en la Constitución de la Organización Mundial de la Salud (OMS), aprobada en 1946 y entrada en vigor en 1948, la «salud es un estado de completo bienestar físico, mental y social, y no sólo consiste en la ausencia de affecciones o enfermedades. La posesión de más alto nivel posible de salud es un derecho fundamental de todos los seres humanos sin distinción de raza, religión, opinión política, económica o condición social. Los gobiernos son responsables de la salud de sus pueblos; puedan hacer frente a esta responsabilidad, buscando las medidas de salud y sociales adecuadas».
}

Sobre la nueva construcción idiomatica de la salud global y de como la progresiva internacionalización de los problemas de salud pública y su interconexión íntima con la ley, la economía y la política sean el resultado del proceso de globalización, lo que tuvo un impacto tanto en la gobernanza de la salud humana como los diversos determinantes de la salud, se vea S. NEGRI, La tutela della salute pubblica internazionale tra governance globale, "sovranità sanitaria" e diritti fondamentali, in Studi in onore di Augusto Sinagra, 2013 , p. 335. Negri afirma que «La rilevanza della salute ai fini del perseguimento di altri obiettivi generali essenziali per la comunità, quali la tutela dei diritti umani, la sicurezza internazionale e lo sviluppo sostenibile, emerge in maniera sempre più evidente nei documenti delle organizzazioni internazionali e negli studi recenti». 
Carta de los Derechos Fundamentales de la Unión Europea de Niza, donde el art.1 dice: «La dignidad humana es inviolable. Debe ser respetada y protegida».

Este mirada reforza «la protección de los derechos fundamentales a la luz de los cambios en la sociedad, del progreso social y del progreso científico y tecnológico": asì, el derecho a la dignidad y la regla del consentimiento informado se colocan en la categoría de los nuevos derechos, que no encuentran referencias explícitas en las Constituciones nacionales de los Estados miembros. En esta perspectiva vale la referencia a la Convención sobre los Derechos Humanos y la Biomedicina, firmada en Oviedo el 4 de abril de 1997 y a la amplia jurisprudencia de los países europeos y terceros, que reconoce el derecho a la autodeterminación del individuo en el campo de la medicina y su principal instrumento jurídico que es el consentimiento informado, configurado como una «condición de legitimidad» de la atención sanitaria.

Sobre la cuestión de la salud reproductiva de las mujeres, el parámetro ineludible es el principio de la libre determinación, como la capacidad de decidir con conciencia y madurez de su propio destino en términos de opciones de salud y reproducción. Por lo tanto, los límites a la libre determinación, sobre todo si se trata de una prohibición absoluta deberán presentar una adecuada y razonablemente justifica por la incapacidad para proteger de otro modo los intereses de igual rango.

\section{EL ABORTO TERAPÉUTICO SEGUN LA LEY ITALIANA.}

El aborto terapéutico - tema fundamental de la salud reproductiva - señala la necesidad de que el sistema legal, en una sociedad compleja, tiene que trabajar en una perspectiva de equilibrio entre los intereses en conflicto.

La cuestión está regulada por la Ley de 22 de mayo de 1978, n. 194, "Normas para la protección social de la maternidad y la interrupción voluntaria del embarazo»: a pesar del intenso debate en torno a su formulación, consta de veintidós artículos que regulan su aplicación ${ }^{10}$.

Esta ley ha establecido que el Estado garantiza el derecho a la procreación consciente y responsable, reconociendo el valor social de la maternidad y protege la vida humana desde su inicio. También coloca la interrupción voluntaria del embarazo fuera de los medios de control de la natalidad; se permite a la mujer - que en Italia tiene la intención de recurrir a las técnicas de aborto dentro de los primeros 90 días de embarazo - de ponerse en contacto con un centro de orientación familiar o con un centro sociosanitario debidamente facultado o, por último, a un médico de elección. Los consultores ayudan a la mujer con el objetivo de aclarar con ella las razones que pueden conducir a la interrupción del embarazo, proponiendo posibles soluciones a los problemas expuestos, ya sean relacionadas con la salud ${ }^{11}$. El médico tiene el deber de llevar a cabo los controles de prevención necesarios, junto con la obligación de probar la intención de la mujer y dar prueba de esta en un documento que encarna su voluntad. La Ley también

${ }^{10}$ C. FLAMIGNI, L'aborto. Storia e attualità di un problema sociale, Bologna, 2008; M. MORI, Aborto e morale, Capire un nuovo diritto, Torino, 2008; A. BRUNELLI, L'interruzione volontaria della gravidanza: come si ostacola l'applicazione di una legge (a contenuto costituzionalmente vincolato), in Scritti in onore di Lorenza Carlassare, a cura di Brunelli - Pugiotto - Veronesi, Napoli, 2009; G. FERRANDO, Grandi prematuri e decisioni di inizio vita, in Nuova giur. civ. comm., 2009, II, p.97.

${ }^{11}$ P. NUVOLONE - A. LANZI, Gravidanza (interruzione della), in Dig. disc. pen., VI, Torino, 1992, p.33. 
establece que para recurrir a la interrupción voluntaria del embarazo deben cumplirse ciertas condiciones básicas bajo las cuales la continuación del mismo, el parto o la maternidad supondrían una grave amenaza para la salud física o mental de la mujer, en relación con su estado de salud o la condición de sus derechos económicos, sociales o familiares, o con las circunstancias en que se produjo la concepción, o debido a la sobrevenida de previsión de anomalías o malformaciones del feto ${ }^{12}$.

En final, la Ley ha establecido que el aborto debe ser realizado por un médico de obstetricia y ginecología en servicio en un hospital general o en uno de los hospitales públicos especializados.

La Ley ordena que la pregunta para la interupción del embarazo se hace personalmente por la mujer. En los casos en que la mujer está bajo la edad de dieciocho años, para la interrupción del embarazo se requiere la aprobación de aquellos que ejercen sobre ella su autoridad o tutela. Además, se establecieron diversas sanciones por quein causa la interrupción del embarazo o el parto prematuro sin el consentimiento de la mujer o sin cumplir con los procedimientos indicados expresamente por la Ley.

\section{EL CONCEPTO DE OBJECIÓN DE CONCIENCIA EN ITALIA.}

El art.9 de la Ley 194/1978 contempla la objeción de conciencia de los trabajadores de salud y afirma que «la objeción de conciencia exime al personal de salud y a quien exercita las actividades auxiliares de cumplir a los procedimientos y a las actividades específicamente y necesariamente diseñadas para determinar la interrupción del embarazo, y no de la asistencia previa y posterior a la intervención».

Esto es lo que la Ley ha establecido para planear en el ordinamiento italiano el contenido de la objeción de conciencia.

El médico puede llegar a ser objetor en cualquier momento, porqué no tiene límite de tiempo preciso ${ }^{13}$ : es decir, si él participa activamente en un procedimiento de aborto (incluso solamente dando el consejo sobre donde ejecutarlo) pierde la condición de objetor de manera irrevocable, lo que resulta en la incapacidad del médico para ejercer de nuevo la objeción.

El Servicio Sanitario Nacional italiano garantiza que los abortos pueden tener lugar en diversos hospitales designados para que, a continuación, si el personal contratado se compone en su totalidad de objetores tendrá que superar esta deficiencia con el fin de garantizar el servicio, por ejemplo, a través de transferencia de personal no objetor.

El médico no puede negarse a intervenir en caso de necesidad, o si la mujer está en peligro de vida (por ejemplo, si una mujer llega con hemorragia grave para un aborto

\footnotetext{
${ }^{12}$ D. CARUSI, La tutela giuridica della vita prenatale e risarcimento del danno nell'illecito plurioffensivo, in Rass.dir.civ., 1992.

${ }^{13}$ G. DALLA TORRE, Diritti dell'uomo e ordinamenti sanitari contemporanei: obiezione di coscienza o opzione di coscienza?, in Realtà e prospettive dell'obiezione di coscienza, a cura di B. Perrone, Milano, 1992 , p.290 s.; V. TURCHI, Obiezione di coscienza, in Dig. disc. priv., XII, Torino, 1995, p.538 ss.; R. NAVARRO VALLS - J. MARTÍNEZ TORRÓN, Le obiezioni di coscienza. Profili di diritto comparato, con la collaborazione di R. Palomino e V. Turchi, Torino, 1995, p.101 ss.
} 
clandestino, el objetor tiene la obligación de llevar a cabo del procedimiento de aborto, si esto es necesario para salvar a la mujer).

En caso de gran adherencia de los profesionales a la objeción de conciencia, la ciudadanía residente puede encontrarse en problemas por la prestación de determinados beneficios, lo que lleva a la pérdida de ciertos derechos: por esta razón, en 2014, Europa ha pedido a Italia de resolver estos problemas.

Si el doctor es objetor, él tiene la obligación de proporcionar su asistencia médica y asegurarse si hay un sustituto. Si una mujer pide un aborto y no lo realiza incluso si la solicitud es de acuerdo con la ley, el médico se carga los riesgos de inhabilitación médica y del despido: la mujer interesada podrá solicitar la reparación del daño biológico.

Este objeción es, de hecho, una acción libre de cualquier responsabilidad personal y cuyas consecuencias recaen por un tercero (en el caso de falta de prestación sanitaria, puede ser el usuario que solicita el servicio al sistema nacional de salud): diversos son los requisitos para poder hablar de la objeción de conciencia ${ }^{14}$.

Sin embargo, esta forma de "argumento" está permitido en todos los Estados del mundo occidental que proporcionan el aborto, salvo en casos de peligro para la vida de la mujer, donde por lo general no se permite la objeción.

Se puede decir que colocar la objeción de conciencia en la Ley sobre la interrupción voluntaria del embarazo parece una contradicción, ya que permite un servicio y al mismo tiempo limita su ejercicio, indicando también las herramientas de su "sabotaje". Lo que significa que si un médico no ayuda a una paciente que quiere hacer un aborto, el incidente se clasifica como una objeción de conciencia y no como un fallo en el servicio público.

Esto, lamentablemente, sucede muy a menudo en los hospitales italianos que no garantizan lo que la ley indica como comportamiento adecuado, es decir, garantizar que las mujeres pueden encontrar - al lado del objetor - un ginecólogo que los permite interrumpir el embarazo.

En 2013, el Tribunal Supremo señaló que la conducta del médico de guardia al cual una paciente pidió ayuda a un paciente para abortar, se abstiene de realizar sus actividades en las etapas anteriores o posteriores a las necesarias a determinar el aborto, invocando el derecho a la objeción de conciencia representa "el delito de negar actos de oficina"15.

Más recientemente, el Consejo de Estado ha establecido que la objeción de conciencia se refiere, por su propia naturaleza, al «foro interior» del individuo y no a lo de una institución pública en su conjunto que, por el contrario, debe prepararse, a pesar del rechazo en la cura de la salud por el profesional individual debido a razones de autonomía profesional y moral, a garantizar la atención médica debida. El Consejo de estado añade que a quien avanza razones de conciencia se puede y se debe argumentar que sólo los

\footnotetext{
${ }^{14}$ A. D'ATENA, Commento all'art. 9, in AA. VV., Commentario alla I. 22 maggio 1978, n. 194, in Le nuove leggi civili commentate, a cura di C. M. Bianca - F. D. Busnelli, I, 1978, 1650 ss.; contra P. MONETA, Obiezione di coscienza II (profili pratici), in Enc. giur., XXI, 1991, p.5; M. P. IADICICCO, Obiezione di coscienza all'aborto ed attività consultoriali: per il T.A.R. Puglia la presenza di medici obiettori nei Consultori familiari è irrilevante, ma non del tutto..., in Giur. cost., 2011, 2, 2000 ss.
}

${ }^{15}$ Corte Cass., sez. VI, 2 aprile 2013, n. 14979. 
individuos tienen una conciencia mientras la conciencia de las instituciones son las leyes que las regulan ${ }^{16}$.

\section{ALGUNAS CONSIDERACIONES FINALES.}

En todo tratamiento o intervención médica respetuosa del principio de la autonomía de la voluntad del paciente hay que garantizar el derecho a la información jurídicosanitaria del que son titulares todos los ciudadanos, pero sin duda es en el terreno de la IVE donde este mandato se hace más necesario que nunca por los importantes intereses en juego que aquí se encierran.

Ante todo tratamiento o intervención, el paciente tiene que disponer de una información jurídico-sanitaria adecuada que le permita, en ejercicio de su autonomía de la voluntad, consentir o rechazar esa actuación médica sabiendo todos los pros y contras que su decisión puede conllevar. Más aún en un terreno de arenas movedizas como el de la IVE, donde hay que ponderar tantos intereses en conflicto y cuando está en juego una vida en formación cuyo futuro es incierto. De este modo, la Administración sanitaria ha de aportar a la mujer (y, en su caso, también a su entorno) información veraz, objetiva, privada de sesgos ideológicos o morales, y que no la instrumentalice. Una información, de carácter multidisciplinar y personalizada de acuerdo con sus intereses ${ }^{17}$.

Los profesionales sanitarios han de abstenerse de imponer aquí criterios de actuación basados en sus propias creencias y convicciones personales, morales, religiosas o filosóficas, han de respetar los valores, creencias y preferencias de estas mujeres en una decisión tan complicada como dolorosa.

Por supuesto, el Estado ha de garantizar los derechos fundamentales de la mujer embarazada (vida, salud, libertad y dignidad) y por esta razón, el derecho a la información de estas mujeres ha de prevalecer sobre un posible derecho a la objeción de conciencia de un médico de familia.

La regulación del aborto ha sido una de las tantas instituciones que - en el siglo pasado -requería una reforma urgente para permitir a las mujeres en recuperar su libertad y alcanzaran la igualdad sustantiva o superar el tiempo de la subordinación. Este discurso - que podemos indicar como de justicia reproductiva - enfrenta el tema central de la salud de la mujer y, segundaria, la cuestion del tipo de regulación administrativa de las instalaciones para la realización de abortos y las normas para la habilitación profesional de la práctica.

Hablar de aborto significa hablar de un derecho que no puede ser interupto por una legislación contraria como la de la objeción de conciencia, que in Italia està planeando problemas concretos de peligro de vida de las mujeres.

La oposición a las políticas de derechos reproductivos, y a las reformas - en Paises que no la tienen - para la liberalización del aborto en particular, se ha identificado a menudo con los grupos organizados que encarnan visiones extremas y conservadoras de la sexualidad de las personas, de los roles de las mujeres y su función reproductiva, entre

\footnotetext{
${ }^{16}$ Cons. Stato Sez. III, 2 settembre 2014, n. 4460.

${ }^{17}$ F. M. BOMBILLAR SAENZ, Tratamiento jurídico del derecho-deber de información en la interrupción voluntaria del embarazo, in Revista da AJURIS, 42, 2015, p.523.
} 
otros. Sin embargo, la resistencia a la reforma del aborto no es el resultado exclusivo de la acción de estos grupos organizados más fundamentalistas. Sucede a menudo que la hostilidad a la aprobación o la aplicación de las reformas sobre los derechos reproductivos es producto también de un "veto moral" que tienden a ejercer estos grupos conservadores.

La dinámica del veto moral y las dificultades históricas para avanzar en la aplicación concreta de un modelo de permisos con implementación es el risultado de la confrontación entre un derecho absoluto a la vida del feto, supuestamente reconocido en la Constitución y los tratados de derechos humanos, y la libertad total de la mujer de disponer de la vida de un tercero. Ello, con la previsible reacción generalizada a favor de la preservación del statu quo frente a lo que se percibe como un planteo de confrontaciónde dos cosmovisiones morales extremas, ha desatado - en Italia - después de la promulgación de la Ley 19 de febrero de 2004 n. 40 "Normas sobre la procreación médicamente asistida", un intenso debate sobre el principio de la vida, con una oposición entre partidarios pro life y pro choice ${ }^{18}$.

Por otra parte, debe también tenerse presente la importancia de que los tribunales logren articular la diferencia entre el interés constitucional en la protección de la vida intrauterina y el reconocimiento de un derecho a la vida de parte del embrión. La comprensión de esta diferencia ha sido un presupuesto básico de las argumentaciones que hoy, en Italia, se llama el vacío - por los tribunales - del contenido de la ley sobre la medicina reproductiva assitita.

\section{BIBLIOGRAFIA}

V. CRISAFULLI, Le norme programmatiche della Costituzione, 1952.

L. CHIEFFI, (a cura), II diritto alla salute alle soglie del terzo millennio. Profili di ordine etico, giuridico ed economico, Torino, 2003.

P. BARILE, Diritti dell'uomo e libertà fondamentali, Bologna, 1981.

A. PACE, Problematica delle libertà costituzionali. Parte generale, Padova, 1992.

C. AMIRANTE, Diritti di libertà e diritti sociali, Napoli, 1995.

L. CARLASSARE, Forma di stato e diritti fondamentali, Quaderni costituzionali, vol.1, 1995.

\footnotetext{
${ }^{18}$ F. D. BUSNELLI, Libertà di coscienza etica e limiti della norma giuridica: l'ipotesi della procreazione medicalmente assistita, in Familia, 2003, p.281; L. d'AVACK, La legge sulla procreazione medicalmente assistita: un'occasione mancata per bilanciare valori ed interessi contrapposti in uno stato laico, in Dir. famiglia, 2004, p.793 ss.; G. FERRANDO, La nuova legge in materia di procreazione medicalmente assistita: perplessità e critiche, in Corriere giur., 2004, p.810 ss.; M. SESTA, Dalla liberta' ai divieti: quale futuro per la legge sulla procreazione medicalmente assistita?, in Corriere giur., 2004, 1528 ss.; R. VILLANI, Dal Parlamento alle aule di giustizia : le nuove disposizioni in materia di procreazione medicalmente assistita al banco di prova del caso concreto, in Nuova giur.civile commentata, n.4, 2004, p.427; P.RESCIGNO, Danno da procreazione, Milano, 2006; E. PELLECCHIA, Aborto farmacologico e disciplina dell'interruzione di gravidanza, in La nuova giur.civ.comm., n.1, 2010 ; P. ZATTI, II duttile rigore : l'approccio di Giorgio Oppo al diritto della vita nascente, in Riv.dir.civ., n.4, 2010 ; A. SCALERA, Aborto. La legge 194 ancora una volta al vaglio della Consulta, in Fam. e diritto, n.11, 2012 ; A. VAPINO, La prova della volontà abortiva ai fini del risarcimento da nascita indesiderata, in Giur.it., n.1, 2015.
} 
F. MODUGNO, I «nuovi diritti» nella giurisprudenza costituzionale, Torino, 1995.

R. GUASTINI, Specificità dell'interpretazione costituzionale?, Analisi e diritto, Torino, 1996.

A. BALDASSARRE, Diritti della persona e valori costituzionali, Torino, 1997.

P. BILANCIA - G. F. PIZZETTI, Aspetti e problemi del costituzionalismo multilivello, Milano, 2004.

P. PERLINGIERI, La persona e i suoi diritti. Problemi del diritto civile, Napoli, 2005.

S. LABRIOLA, (a cura di), Valori e principi del regime repubblicano. 3. Legalità e garanzie, Roma-Bari, 2006, p.451 ss.; Id, Piccola storia dell'attualità della Costituzione, Milano, 4, 2006.

D. MORANA, La salute nella Costituzione italiana, Milano, 2002.

F. MANTOVANI, II consenso informato: pratiche consensuali, in Riv.it.med.leg., 2000.

M.C. VENUTI, Gli atti di disposizione del corpo, Milano, 2002.

S. CACACE, Informazione, consenso e rifiuto di cure. (il)liceità del trattamento sanitario, in comande' (a cura di), Diritto privato europeo e diritti fondamentali, Torino, 2004.

C.M. D'ARRIGO, II contratto e il corpo: meritevolezza e liceità degli atti di disposizione dell'integrità fisica, in Familia, 2005.

G. ALPA, II principio di autodeterminazione e le direttive anticipate sulle cure mediche, in Riv.crit.dir.priv., 2006.

G. FACCI, Violazione del dovere di informazione da parte del sanitario e risarcimento del danno, in Resp.civ. e prev., 2006.

G. FERRANDO, Stato vegetativo permanente e sospensione dei trattamenti medici, in Testamento biologico. Riflessioni di dieci giuristi, Fondazione Umberto Veronesi, 2006.

A. PINNA, Autodeterminazione e consenso: da regola per i trattamenti sanitari a principio generale, in Contr.e impr., 2006.

S. RODOTA', La vita e le regole. Tra diritto e non diritto, Milano, 2006.

L. VIOLINI, A. OSTI, Le linee di demarcazione della vita umana, in M. Cartabia (a cura di), I diritti in azione. Universalità e pluralismo dei diritti fondamentali nelle Corti europee, Bologna, 2007.

P. ZATTI, II corpo e la nebulosa dell'appartenenza, in Nuova giur.civ.comm., 2007.

G. CRICENTI, I diritti sul corpo, Napoli, 2008.

L. D'AVACK, Sul consenso informato all'atto medico, in II dir.fam. e pers., 2008.

D. MESSINETTI, L'autodeterminazione dispositiva della persona e il valore della libertà del soggetto, in Riv.crit.dir.priv., 2008.

G. MONTANARI VERGALLO, II rapporto medico paziente. Consenso e informazione tra libertà e responsabilità, Milano, 2008.

G.U. RESCIGNO, Dal diritto di rifiutare un determinato trattamento sanitario secondo l'art.32, co. $2^{\circ}$ Cost., al principio di autodetermin azione intorno alla propria vita, in Dir.pubbl., 2008. 
D. CARUSI, Tutela della salute, consenso alle cure, direttive anticipate: l'evoluzione del pensiero privatistico, in Riv.crit.dir.priv., 2009.

C. CASONATO, Introduzione al biodiritto, Torino, 2009.

T. PASQUINO, Autodeterminazione e dignità della morte. Saggio di diritto civile, Padova, 2009.

B. SALVATORE, Per uno studio sul consenso informato, in Dir.giur., 2009.

U. CARNEVALI, Omessa informazione da parte del medico, danno da trattamento terapeutico e ipotetica scelta del paziente, in Resp.civ.e prev., 2010.

C. CASTRONOVO, Autodeterminazione e diritto privato, in Europa e diritto privato, 2010.

G. MARINI, II consenso, in Trattato di biodiritto, diretto da S. Rodotà e P.Zatti, Ambito e fonti del biodiritto, a cura di S.Rodotà e M.Tallacchini, Milano, 2010.

F.D. BUSNELLI, problemi giuridici di fine vita tra natura ed artificio, in Riv.dir.civ., 2011.

G. CRICENTI, Diritto all'autodeterminazione? Bioetica dell'autonomia privata, in Nuova giur.civ.comm., 2011.

G.RESTA, La disposizione del corpo. Regole di appartenenza e di circolazione, in Trattato di biodiritto, diretto da S.Rodotà e P.Zatti, II governo del corpo, I, Milano, 2011.

S. STEFANELLI, Autodeterminazione e disposizioni sul corpo, Perugia, 2011.

E. CAVASINO, La flessibilità del diritto alla salute, Napoli, 2012.

S. RODOTA', II diritto di avere diritti, Roma-Bari, 2012.

V. IVONE, Vulnerabilità del corpo e diritto al consenso, Napoli, 2013.

S. PUGLIATTI, L'atto di disposizione e il trasferimento dei diritti, in Diritto civile, Metodo, teoria, Pratica. Saggi, Milano, 1951.

M. PARADISO, II dovere del medico di informare il paziente. Consenso contrattuale e diritti della persona, in A.A.V.V., La responsabilità medica, Milano, 1982.

F. GALGANO, Contratto e responsabilità contrattuale nell'attività sanitaria, in Riv. trim. dir. proc. civ., 1984, p.721.

M. COSTANZA, Informazione del paziente e responsabilità del medico, in Giust.civ., 1986.

L. ROSSI CARLEO, Brevi considerazioni sulla problematica della forma del consenso negli atti di disposizione del corpo, in La forma degli atti di diritto privato. Studi in onore di M.Giorgianni, Napoli, 1988.

G. GRISI, L'obbligo precontrattuale di informazione, Napoli, 1990.

R. ROMBOLI, Limiti alla libertà di disporre del proprio corpo nel suo aspetto "attivo" e in quello "passivo", in Foro it., 1991.

R. DE MATTEIS, La responsabilità medica: un sottosistema della responsabilità civile, Padova, 1995.

A. SANTOSUOSSO, II consenso informato, Milano, 1996.

C. CASTRONOVO, Profili della responsabilità medica, in Studi in onore di Pietro Rescigno, V, Milano, 1998. 
G. FERRANDO, Consenso informato del paziente e responsabilità del medico. Principi, problemi e linee di tendenza, in Studi in onore di Pietro Rescigno, 2000.

A. DONATI, Consenso informato e responsabilità da prestazione medica, in Rass.dir.civ., 2000.

M. CARTABIA, I diritti fondamentali in Europa dopo Lisbona: verso nuovi equilibri?, in Giornale dir. amm., 2010.

V. SCALISI, Ermeneutica dei diritti fondamentali e principio "personalista" in Italia e nell'Unione Europea, in Riv. dir. civ., 2010.

S. NEGRI, Transplant Ethics and the International Crime of Organ Trafficking, in International Criminal Law Review, 1, 2016.

S. NEGRI, La tutela della salute pubblica internazionale tra governance globale, "sovranità sanitaria" e diritti fondamentali, in Studi in onore di Augusto Sinagra, 2013.

C. FLAMIGNI, L'aborto. Storia e attualità di un problema sociale, Bologna, 2008.

M. MORI, Aborto e morale, Capire un nuovo diritto, Torino, 2008.

A. BRUNELLI, L'interruzione volontaria della gravidanza: come si ostacola l'applicazione di una legge (a contenuto costituzionalmente vincolato), in Scritti in onore di Lorenza Carlassare, a cura di Brunelli - Pugiotto - Veronesi, Napoli, 2009.

G. FERRANDO, Grandi prematuri e decisioni di inizio vita, in Nuova giur. civ. comm., 2009.

P. NUVOLONE - A. LANZI, Gravidanza (interruzione della), in Dig. disc. pen., VI, Torino, 1992.

D. CARUSI, La tutela giuridica della vita prenatale e risarcimento del danno nell'illecito plurioffensivo, in Rass.dir.civ., 1992.

G. DALLA TORRE, Diritti dell'uomo e ordinamenti sanitari contemporanei: obiezione di coscienza o opzione di coscienza?, in Realtà e prospettive dell'obiezione di coscienza, a cura di B. Perrone, Milano, 1992.

V. TURCHI, Obiezione di coscienza, in Dig. disc. priv., XII, Torino, 1995.

R. NAVARRO VALLS - J. MARTíNEZ TORRÓN, Le obiezioni di coscienza. Profili di diritto comparato, con la collaborazione di R. Palomino e V. Turchi, Torino, 1995.

A. D'ATENA, Commento all'art. 9, in AA. VV., Commentario alla I. 22 maggio 1978, n. 194, in Le nuove leggi civili commentate, a cura di C. M. Bianca - F. D. Busnelli, I, 1978.

P. MONETA, Obiezione di coscienza II (profili pratici), in Enc. giur., XXI, 1991.

M. P. IADICICCO, Obiezione di coscienza all'aborto ed attività consultoriali: per il T.A.R. Puglia la presenza di medici obiettori nei Consultori familiari è irrilevante, ma non del tutto..., in Giur. cost., 2011.

F. M. BOMBILLAR SAENZ, Tratamiento jurídico del derecho-deber de información en la interrupción voluntaria del embarazo, in Revista da AJURIS, 42, 2015.

F. D. BUSNELLI, Libertà di coscienza etica e limiti della norma giuridica: l'ipotesi della procreazione medicalmente assistita, in Familia, 2003. 
L. d'AVACK, La legge sulla procreazione medicalmente assistita: un'occasione mancata per bilanciare valori ed interessi contrapposti in uno stato laico, in Dir. famiglia, 2004.

G. FERRANDO, La nuova legge in materia di procreazione medicalmente assistita: perplessità e critiche, in Corriere giur., 2004.

M. SESTA, Dalla liberta' ai divieti: quale futuro per la legge sulla procreazione medicalmente assistita?, in Corriere giur., 2004.

R. VILLANI, Dal Parlamento alle aule di giustizia : le nuove disposizioni in materia di procreazione medicalmente assistita al banco di prova del caso concreto, in Nuova giur.civile commentata, n.4, 2004.

P.RESCIGNO, Danno da procreazione, Milano, 2006.

E. PELLECCHIA, Aborto farmacologico e disciplina dell'interruzione di gravidanza, in La nuova giur.civ.comm., n.1, 2010.

P. ZATTI, II duttile rigore : I'approccio di Giorgio Oppo al diritto della vita nascente, in Riv.dir.civ., n.4, 2010.

A. SCALERA, Aborto. La legge 194 ancora una volta al vaglio della Consulta, in Fam. e diritto, 11, 2012.

A. VAPINO, La prova della volontà abortiva ai fini del risarcimento da nascita indesiderata, in Giur.it., n.1, 2015. 